\title{
Les mots de l'expérience ou l'expérience des mots. Lire pour écrire des récits de fiction à l'école élémentaire française
}

\author{
Brigitte Marin \\ Université Paris-Est, EA 4384 Circeft-Escol, Université Paris 8, UPEC, 94010 Créteil Cedex, France
}

\begin{abstract}
Résumé. Cette étude longitudinale a pour objectif de montrer l'effet des apprentissages lexicaux sur l'utilisation d'un vocabulaire spécifique en situation d'écriture scolaire. Dans le cadre de cette expérimentation, 432 élèves français de $3^{\mathrm{e}}, 4^{\mathrm{e}}$ et $5^{\mathrm{e}}$ année de l'école élémentaire ont écrit plus de 2000 textes narratifs en langue maternelle sur une thématique donnée. Ces écrits ont été suivis de la lecture de textes d'auteurs portant sur le même thème, à cinq reprises, au cours de l'année scolaire.

Dans un premier temps, les élèves ont écrit un texte, puis ils ont eu accès à différents types de ressources lexicales, avant de réviser ce texte. Chaque session d'écriture incluait un dispositif fondé sur différentes approches cognitives (Lewis, 1993). Les élèves pouvaient s'appuyer sur les explications données par le professeur, pour développer leur texte. Par ailleurs, différentes sortes d'aides lexicales étaient fournies, parmi lesquelles les élèves pouvaient choisir : (i) synonymes de mots usuels, (ii) tableaux de mots concernant le thème de la rédaction - organisés par catégorie grammaticale - et (iii) textes d'écrivains portant sur le même thème.

D'après notre hypothèse, l'aide la plus efficace dépend du niveau des élèves, en lien avec leurs connaissances initiales et leurs compétences de lecture.

Nous avons comparé l'évolution des compétences de lecture et d'écriture d'élèves appartenant à huit groupes expérimentaux, en parallèle avec des élèves appartenant à huit autres groupes travaillant de manière traditionnelle.

Les résultats montrent que les premières analyses, quantitative et qualitative, confirment notre hypothèse. Ils indiquent l'amélioration des compétences liées aux situations où l'apprentissage est planifié par les chercheurs (Gagnon \& Ziarko, 2012), à partir d'activités lexicales orales et écrites (Sardier, 2012).
\end{abstract}

\begin{abstract}
This longitudinal study aims at showing the impact of lexical apprenticeship on the use of specific vocabulary while writing at school. For the experiment, 432 French students in grades 3, 4 and 5 wrote a total of more than 2000 narrative texts in L1 about a specific theme. These writings were following the reading of authors' literary texts about the same subject, five times over the course of the school year. First, the students wrote texts, then they had access to different kinds of lexical resources, and they lastly revised their texts. Each session included a disposal based on different cognitive approaches (Lewis, 1993). All students could take benefit of the teacher's explanations about new words that could be useful to expand their writings. Otherwise, different kinds of lexical helps were provided, between which they could choose : (i) synonyms of ordinary words, (ii) tables with words in connection with the theme of the writing - organized according to their grammatical category - and (iii) texts of authors about the same subject.

Our hypothesis was that the most efficient help was dependent on the level of students, in connection with their prior knowledge and their ability to read. We have compared the
\end{abstract}

This is an Open Access article distributed under the terms of the Creative Commons Attribution License 4.0, which permits unrestricted use, distribution, and reproduction in any medium, provided the original work is properly cited. 


\section{SHS Web of Conferences}

evolution of lexical and writing skills of students belonging to eight experimental groups, in parallel with students belonging to eight other groups having worked in a traditional way. Findings indicate that the first quantitative and qualitative analyses confirm our hypothesis. They show the improvement of skills bound to the situations when lexical apprenticeship are planned by researchers (Gagnon \& Ziarko, 2012), in interaction between verbal and writing activities (Sardier, 2012).

«J'écris toujours mes textes à la main puisque je les rature beaucoup. Ensuite, il est essentiel que je les transcrive moi-même à la machine à écrire parce que vient alors une seconde vague de corrections, allant toujours dans le sens de l'ellipse ou de la suppression. C'est le moment où ce que l'on a écrit, qui reste très subjectif dans l'apparence graphique de l'écriture manuelle, s'objective : ce n'est pas encore un livre ou un article mais, grâce aux caractères de la machine à écrire, il y a déjà une apparence objective et c'est une étape très importante. »

Roland Barthes, Le grain de la voix. Entretiens

\section{Introduction}

Des mots de l'expérience à l'expérience des mots, se dessinent en contrepoint les parcours et les finalités d'écriture, de l'écrivain novice et de l'écrivain expert. Deux traits les rassemblent dans leurs dissemblances, comme dans leurs ressemblances, si l'on se réfère à la pratique scripturale de Roland Barthes [1] : le processus d'ellipse vs. d'expansion lors de la révision de texte et le rapport à la typographie. En effet, pour l'élève de la fin de l'école élémentaire, les mots issus de l'expérience scolaire complètent une première traduction de l'expérience du monde et viennent s'ajouter à leurs ressources lexicales initiales. La récriture vise alors à densifier le texte par le processus de l'ajout. En revanche, certains professionnels de l'écriture ont tendance à recourir à une poétique de la concision. Le second point de confrontation, de ressemblance, cette fois, concerne la manière dont la textualisation s'officialise ou s'objective par la transposition de l'écriture manuelle dans le corps typographique d'une police de caractères. L'impression du texte renouvelle l'impression sur le texte, quel qu'en soit l'auteur, expert ou novice, qui en devient le lecteur averti.

Ces différents processus entrent en jeu dans l'étude qui fait l'objet de cet article où la question de l'enseignement du vocabulaire occupe une place prééminente.

Cette recherche s'inscrit résolument dans une approche pragmatique de mise en lien entre recherche et formation, telle que les induisent les enjeux sociaux liés à la création des écoles supérieures du professorat et de l'éducation (ESPÉ) dans le contexte français de la formation des enseignants. Il s'agit de tester un dispositif didactique élaboré pour les besoins de la recherche en phase avec les programmes scolaires mis en œuvre dans les classes, en cohérence avec la politique globale de réduction des inégalités entre élèves, dont la nécessité a été mise en exergue par les résultats des enquêtes PISA [2]. La réflexion porte sur la mobilisation en situation des mots étudiés et la construction de ressources pour la formation.

Le lexique constitue en effet un pôle important de différenciation dans l'acquisition et la maîtrise des savoirs et ce faisant de la réussite scolaire. Le répertoire lexical des élèves influence les premiers apprentissages et l'entrée dans l'écrit, qui eux-mêmes déterminent la suite de leur curriculum. Le lexique des élèves est constitué d'un capital de mots acquis et mobilisables dans le discours, alors que le lexique de l'écrit fait essentiellement intervenir des termes extérieurs à l'univers langagier de nombreux élèves en situation d'échec scolaire [3]. Les élèves qui ont de faibles ressources lexicales à l'écrit éprouvent donc des difficultés de compréhension [4] des attendus linguistiques et langagiers de l'école, ce qui pose la question de ses stratégies d'enseignement et d'acquisition.

Dans le cadre de cet article, nous nous intéressons à la dimension lexicale des apprentissages de la fin de l'école primaire. À la charnière entre les premiers apprentissages et le passage au collège - où se spécifient les savoirs lexicaux en fonction des disciplines et des genres de textes - l'enseignement du vocabulaire nécessite une attention particulière et requiert la mise en œuvre de dispositifs testés expérimentalement. 
Nous présenterons l'objectif de la recherche, la méthodologie utilisée, puis une analyse de production d'élève en situation expérimentale, avant de procéder à l'interprétation des résultats obtenus.

\section{Objectifs de la recherche}

Le vocabulaire de l'écrit n'est pas couramment mobilisé dans les classes, où communication et échanges entre enseignant et élèves relèvent principalement de la langue orale. Quand il s'agit d'enrichir le vocabulaire dans les classes de cycle 3, les activités lexicales concernent le plus souvent la mobilisation des mots pris isolément alors que pour les apprendre, il faut les faire exister en discours. Les activités les plus fréquentes mettent en œuvre des stratégies d'acquisition fondées sur des activités spécifiques de structuration du vocabulaire ou de mémorisation de listes ou de familles de mots d'après leurs traits sémantiques, et fondées sur une conception behavioriste. Ces manipulations linguistiques se limitent souvent à la prise en compte des relations d'observations morphologiques (radical et affixes) parfois complétées par des recherches dans le dictionnaire.

En rupture par rapport à ces procédures, le protocole expérimental que nous avons testé a été établi à partir de constats enseignants récurrents : les activités de vocabulaire autonomes - ou décrochées des autres activités d'enseignement de la langue - ne conduisent que partiellement au réinvestissement en situation d'écriture des mots nouveaux récemment appris. C'est pourquoi la méthodologie adoptée consistait à tester l'hypothèse selon laquelle la concomitance entre apparition et réemploi de mots nouveaux en contexte favorise leur assimilation [5]. La deuxième hypothèse concernait l'effet des différentes modalités d'accès à un vocabulaire en phase avec une situation d'écriture permettant sa réutilisation.

Ainsi cette recherche concerne plus particulièrement le processus de mémorisation de mots inconnus ou peu connus, relevant du vocabulaire passif, en lien avec leur contexte d'émergence et l'appareil textuel $^{1}$ (oral ou écrit) qui leur fait progressivement prendre sens au fil des explications ou des aides méthodologiques fournies par l'enseignant. L'élaboration du dispositif expérimental s'est appuyée sur l'opérationnalisation de plusieurs types d'aides.

\section{Les aides verbales}

Parmi les ressources proposées aux élèves figuraient en premier lieu les explications de l'enseignant sur les mots rencontrés dans les textes d'auteurs lus en classe. Ces mots étaient analysés collectivement et glosés par l'expérimentatrice ${ }^{2}$ - qui prenait le relais de l'enseignant habituel de la classe pendant le temps des séquences élaborées et proposées, en lien avec les retombées attendues ultérieurement sur l'évolution des pratiques de classe.

Le choix de la même enseignante expérimentatrice pour l'ensemble des séquences des élèves du groupe expérimental, coûteux en temps ${ }^{3}$, a permis d'éviter les biais liés à l'effet-maître, et par conséquent, permis de constituer un corpus plus cohérent que n'aurait conduit à le faire un protocole confié à l'enseignant de chaque classe ${ }^{4}$.

\footnotetext{
${ }^{1}$ Nous donnons ici au mot texte le sens global à la fois du texte oral produit par l'enseignant (le texte de l'enseignant), comme son sens le plus courant de texte écrit. Ce texte se présente sous une forme brève (telle une définition de dictionnaire) ou une forme longue (tel un texte d'auteur).

2 Afin de donner une plus grande robustesse aux résultats de notre étude, une enseignante maître-formatrice impliquée dans la recherche, Annick Cautela a mis en œuvre les séances de classe dans l'ensemble des classes expérimentales, pendant deux années consécutives, 2010-2011 et 2011-2012.

3 J'adresse ici mes plus vifs remerciements à Annick Cautela pour son investissement à tous les niveaux de cette recherche, de l'élaboration à la mise en œuvre et à l'analyse des résultats.

${ }^{4}$ En revanche, dans les classes témoins, les enseignants ont laissé libre cours aux pratiques d'enseignement du vocabulaire qui leur étaient coutumières.
} 
Pour ce qui est des commentaires oraux de l'expérimentatrice, la mise en œuvre du protocole était fondée sur une visée précise, la mémorisation et l'appropriation du vocabulaire enseigné aux élèves. La réflexion approfondie conduite systématiquement sur les signifiant et signifié des nouveaux mots introduits était destinée à y contribuer.

\section{Les aides écrites}

Parmi les aides écrites ont été fournis, d'une part des outils proposant des aides lexicales ponctuelles, ciblées, présentées sous forme de listes raisonnées, mais également sous une forme tabulaire [Annexes A, B, C], et d'autre part, des textes d'écrivains pour la jeunesse.

Les « réservoirs de mots » disponibles dans les tableaux de mots étaient organisés par catégorie sémantique et grammaticale. Ils conduisaient ainsi les élèves à prélever parmi l'ensemble de ces unités lexicales, indépendantes les unes des autres, l'élément cohérent sur ces deux plans pour l'insérer dans leur propre texte et, éventuellement le développer à partir de ces déclencheurs d'expansions.

Les textes d'auteurs favorisaient une stratégie plus complexe [6], requérant de sélectionner dans le texte lu les éléments lexicaux ou phrastiques entrant en cohérence avec la tonalité du texte initial rédigé par l'élève et pouvant y être intégrés harmonieusement, sans rupture énonciative ou stylistique. Le nombre de processus associés à ce travail de sélection, puis d'insertion lexicale le rendait plus exigeant sur le plan cognitif [7]. Dès lors s'imposait une troisième hypothèse de recherche concernant le choix et l'effet des aides lexicales sur l'amélioration des productions de texte en fonction du niveau des élèves.

\section{Méthodologie}

\section{Mise en place de l'expérimentation}

Afin de pouvoir apprécier le développement des compétences lexicales des élèves en lien avec le dispositif qui sera décrit infra, nous avons testé leurs compétences initiales en début d'année pour mesurer l'écart avec celles qu'ils manifestaient en fin d'année. Dans l'intermittence ont été mises en place les séances d'apprentissage lexical construites pour les besoins de la recherche, en appui sur les activités d'enseignement de la langue relevant des programmes.

Le dispositif a ainsi consisté en (i) un prétest d'écriture en septembre (rédaction d'une suite de texte); (ii) trois séquences d'apprentissage en octobre, décembre, avril ; (iii) un posttest en mai (suite de texte donnant lieu à la comparaison avec les classes témoins).

453 élèves ont participé à l'ensemble des séances de travail. Ils étaient issus de douze classes expérimentales et de six classes témoins. Les élèves des classes témoins étaient soumis au même cahier des charges que les élèves des classes expérimentales : production d'une première version d'un texte narratif faisant suite à un texte déclencheur lu en classe, puis d'une seconde version. Cependant, les différences d'activité entre classes témoins et classes expérimentales portaient sur l'environnement d'apprentissage. Il était laissé libre cours à la pratique enseignante habituelle à chaque enseignant dans la première situation, $v s$. il était l'objet d'un dispositif construit et étayé sur des cadres de référence théorique et des hypothèses de recherche, dans la seconde.

\section{Protocole expérimental}

Le protocole expérimental était identique pour les trois niveaux de classe concernés par l'expérimentation : troisième, quatrième et cinquième année de l'école élémentaire. Pour le décrire en situation nous prendrons des illustrations dans le corpus constitué par le matériel et le corpus de la troisième année de l'école élémentaire.

Au cours de la première séquence d'apprentissage lexical, lors de la séance 1 , les élèves ont lu un texte court (un extrait de L'oiseau d'or ${ }^{5}$ ). Ensuite, l'expérimentatrice a procédé à un questionnement

${ }^{5}$ Mouloud Mammeri, Contes berbères de Kabylie, Pocket Jeunesse, 2000. 
maïeutique sur le sens des mots difficiles. A suivi une première version d'écriture. Puis la séance 2 a proposé la lecture critique et récriture instrumentée des premières versions ainsi que la présentation d'outils d'aide à la récriture (listes de mots ou expressions fabriqués par les enseignants-chercheurs, courts textes d'auteurs, familiarisation à la récriture à partir des outils présentés). Puis il a été demandé la récriture par chaque élève de son texte initial.

La séance de récriture s'est appuyée sur un second texte ressource, Dans la forêt profonde ${ }^{6}$. D' autres outils d'aide à la récriture sont proposés, telle une liste d'expressions idiomatiques variées, comme, par exemple : «n'écoutant que son bon cœur ${ }^{7}$ » ou «bondit de joie» [Annexe A].

Parmi ces outils figurent également des pages du dictionnaire des synonymes [Annexe B] proposant des formulations différentes destinées à varier l'expression, mais aussi à en préciser les nuances en fonction de la situation d'énonciation, des registres de langue et des effets de style recherchés. Ces « réservoirs de synonymes » offrent des ressources portant aussi bien sur les verbes introducteurs de discours, que les verbes de mouvement, ou les adjectifs qualificatifs permettant de caractériser les personnages et de planter le décor en favorisant l'expression des nuances permises par les choix proposés. Dire ou rétorquer n'a pas le même sens ; l'usage de tel ou tel mot n'a pas le même effet sur la clarification de l'intention que le scripteur prête au personnage à qui il donne vie par les mots qu'il lui prête.

Pour enrichir et diversifier les ressources mises à disposition des élèves, une grille thématique de ressources lexicales [Annexe C] proposait des entrées grammaticales (noms et groupes nominaux, adjectifs qualificatifs, verbes et adverbes) et thématiques (concernant le héros, l'héroïne, leurs actions, le cadre spatial... ). Ces entrées croisées favorisent les choix des mots permettant d'enrichir les productions textuelles initiales en jouant simultanément sur l'axe paradigmatique et syntagmatique. La quatrième ressource proposée consistait en un texte d'auteur de littérature de jeunesse sur le même thème (cf. note 6).

Par ailleurs, à la fin de la séance de récriture, un questionnaire succinct a été soumis aux élèves [Annexe D] afin de recueillir des données sur les aides auxquelles ils s'étaient référés et dont ils avaient estimé avoir tiré profit.

\section{Analyse qualitative des textes produits par les élèves}

Nous aborderons ici le volet qualitatif de l'étude, préalablement à une analyse quantitative dont les résultats, en cours de traitement, dessinent une tendance à l'accroissement du volume des textes. Cette augmentation concerne en particulier l'ajout de mots ancrés sémantiquement dans la tonalité du récit.

Sur le plan qualitatif, le développement du vocabulaire est plus nettement perceptible dans des situations d'écriture en phase avec le travail lexical en situation, ce qui correspond à la validation de notre première hypothèse. Ce premier résultat issu d'entretiens avec les enseignants des classes expérimentales comme des classes témoins reste à confirmer par des analyses quantitatives.

En revanche, l'amélioration de la qualité des textes produits entre les deux versions d'écriture, du début à la fin de l'année, s'avère plus probante pour les élèves des classes expérimentales ayant bénéficié d'aides lexicales différentes, laissées à leur initiative. Pour donner un exemple représentatif des constats effectués par les expérimentateurs comme par les enseignants des classes, nous retracerons l'effet des différentes aides lexicales proposées à partir des strates d'écriture successives d'un élève ${ }^{8}$.

L'analyse des textes d'élèves s'est appuyée sur les modifications effectuées de la première à la deuxième version, et plus particulièrement sur les emprunts issus des textes sources, du discours de

\footnotetext{
6 Anthony Browne, La Forêt profonde, L'École des loisirs, 2006.

${ }^{7}$ Les différentes annexes [A], [B], [C] renvoyées en fin d'article donnent accès aux différentes catégories de ressources proposées pour une même séquence d'apprentissage lexical associée à une situation d'écriture/récriture.

${ }^{8}$ Dans le cadre de cette étude, nous nous intéressons plus particulièrement aux résultats obtenus dans les classes de début de cycle 3 .
} 
l'enseignant, du dictionnaire des synonymes et de la grille figurant en annexes. Ces modifications opérées lors de la version 2 apparaissent en gras ; elles concernent des ajouts, des suppressions et des substitutions. Les ajouts en caractère gras, ombrés, correspondent à des mots empruntés aux tableaux de mots, alors que les mots ajoutés en gras, soulignés de deux traits, sont issus des explications et des gloses de l'expérimentatrice sur les mots inconnus ou relevant du vocabulaire passif des élèves. Ces mots ont fait l'objet d'éclairages étymologiques et sémantiques visant à en livrer le sens et les sens en langue et en discours.

L'exemple infra permet de suivre les évolutions lexicales liées à l'utilisation des différentes ressources mises à disposition.

Exemple d'un texte d'élève de la classe expérimentale - Séquence 1 - Texte révisé ${ }^{9}$ (version 2).

"Aziza traverse le désert et trouva un scorpion qui essayait de (le) la piquer. Elle le (coupa) $\underline{\underline{\text { transperça }}}^{10}$ avec son couteau. Après elle alla dans la forêt et trouva un groupe de loups qui essayaient de la mordre, ensuite elle vit l'oiseau d'or. Il était bien doré. Il était aussi mélodieux ${ }^{11}$ et chatoyant mais très maléfique et très attirant. Ensuite elle (vit) aperçut ${ }^{12}$ son frère, elle était désespérée. Elle ne pouvait rien faire, alors elle fit un piège captura l'oiseau d'or et obligea l'oiseau à sauver son frère. $[. .]$.

Les ajouts ombrés mettent en évidence la facilité de l'élève à prélever dans les tableaux de mots le signifiant qui permet de donner plus d'emphase à son récit en l'ornant de mots précis comme le verbe apercevoir au lieu du banal voir. Les mots ajoutés d'apparence précieuse, tels que « chatoyant », « mélodieux » ou « maléfique » renvoient à l'esthétique du signifiant qui les fait mémoriser, comme l'ont indiqué les entretiens conduits avec les élèves quelques semaines après l'expérimentation.

Les adjectifs 《 attirant » et « désespérée », quant à eux, repris des explications orales de l'expérimentatrice, traduisent bien le travail langagier rigoureux qui a été mené pour faire comprendre la tension dialectique entre l'attrait maléfique de l'oiseau et le désespoir auquel conduit le sortilège pour quiconque tombe dans son piège. De même l'appropriation du verbe transpercer commenté oralement dans ce contexte révèle la compréhension fine du processus affixal : trans-percer, pour lequel les élèves ont parfaitement perçu le rôle du préfixe dans l'image créée.

La confirmation de nos hypothèses (enrichissement du vocabulaire fondé sur la mobilisation des différentes ressources lexicales proposées en fonction du niveau des élèves) est validée par l'analyse qualitative des textes du corpus. Elle permet de montrer l'effet positif sur les apprentissages lexicaux des listes de mots rationalisées et de l'intervention de l'expérimentatrice lors des moments collectifs, où celle-ci recentre l'activité des élèves sur des processus cognitifs favorisant la mémorisation et par conséquent, l'appropriation du vocabulaire.

C'est ce qu'indiquent les opérations de révision, lesquelles montrent que les ajouts et les substitutions ont pour origine tantôt le discours de l'enseignant (« attirant », « désespérée », « transperça »), tantôt le recours aux grilles de mots (« mélodieux », « chatoyant », « maléfique », « aperçut »).

Par ailleurs, la manière dont les élèves analysent l'utilisation qu'ils ont faite des différentes ressources lexicales proposées [Annexe D] indique des disparités dans les choix qu'ils ont opérés en fonction de leur niveau. On constate ainsi que les élèves en difficulté puisent dans le répertoire lexical de l'enseignant ou des grilles à disposition, alors que les élèves les plus performants s'inspirent des textes d'auteurs.

\footnotetext{
9 Texte d'Alix. Le prénom de l'élève a été modifié pour préserver l'anonymat.

10 Emprunt au discours de l'enseignant.

${ }^{11}$ Emprunt à la grille thématique.

12 Substitution.
} 


\section{5. Éléments de discussion}

Dans le dispositif mis en place, la première des aides lexicales a concerné la glose des mots inconnus identifiés dans le texte d'auteur qui a servi de lanceur à l'activité de production de texte. La polysémie constitue l'une des principales difficultés lexicales. Les mots ont en effet une pluralité de sens liés au contexte. Il est donc important d'entraîner les élèves à établir le lien de cohérence qui unit les polysèmes et les traits sémantiques qui entrent dans leur composition. Par exemple, le verbe marcher, régulièrement utilisé, possède quatre polysèmes qui renvoient, pour un être animé, à deux traits sémantiques, la capacité de se déplacer et celle de faire fonctionner ses membres inférieurs de manière satisfaisante (je marche) et pour un objet technique, au seul fait de fonctionner correctement (ma montre marche). Dire qu'une affaire marche signifie qu'elle suit un cours favorable : le deuxième trait sémantique est conservé tout comme dans « il a marché » où le sujet a fait ce qu'on attendait de lui. Ces polysèmes opèrent par métaphores successives, parfois difficiles à percevoir. Faire découvrir, aux élèves ces glissements du sens d'un même mot dans des emplois hétérogènes leur permet de dépasser la conception monosémique et le rapport homologique entre le mot et son référent pour comprendre l'évolution et la stratification sémantique des mots [8].

C'est l'approche qui a été adoptée par l'expérimentatrice avec l'objectif d'animer les mots et leurs différents sens en langue et en discours. Tout comme l'étymologie qui permet d'éclairer le sens des mots en diachronie ${ }^{13}$, les procédés de structuration du lexique donnent accès à la dynamique linguistique en synchronie.

Parmi les aides écrites et formalisées proposées, les tableaux de mots organisés par thème et par catégorie grammaticale ont été le plus utilisés par les élèves qui y ont puisé des termes adaptés à leurs besoins lexicaux immédiats. Ils ont d'emblée identifié les mots dont le signifiant leur était agréable ; la mémorisation en a été facilitée pour cette raison. Les entretiens postérieurs à l'expérimentation ont révélé cet intérêt pour le mot rare ou précieux.

Le recours aux textes d'auteurs, moins souvent sollicités, est dû à plusieurs facteurs, dont le temps de lecture et les stratégies associées des élèves pour prélever les mots nécessaires à l'amélioration de leur texte initial dans un temps compatible avec l'activité de récriture. Cette question de la temporalité consubstantielle à celles de la fluidité de la lecture et de la compétence à hiérarchiser les informations ressortit à la complémentarité des aides lexicales à proposer en cycle 3. Le choix d'un exemple pris en classe de cours élémentaire deuxième année a été particulièrement éclairant de ce point de vue. En effet, fort peu d'élèves ont tiré profit des textes ressources, alors qu'à la fin de l'école élémentaire, ils ont été moins rares à s'inscrire dans cette procédure. La maîtrise plus affirmée des processus de bas niveau le décodage - leur permet d'allouer davantage de ressources cognitives à la sélection des informations lexicales utiles à la tâche prescrite.

\section{Conclusion}

Si les supports d'apprentissage lexical décrits contribuent, de diverses manières et à différents niveaux [9], à l'acquisition de compétences narratives, la lecture de textes en réseau reste essentielle au développement du répertoire lexical. Celle-ci constitue une source de découverte et de réinvestissement de mots d'un même domaine référentiel.

En écho à l'épigraphe de Roland Barthes, il n'est sans doute pas inutile de rappeler que les textes rédigés par les élèves ont été dactylographiés et mis en ligne sur un site web, ce qui a permis de les diffuser largement et de les faire ainsi accéder à un lectorat plus vaste que celui ordinairement constitué par le seul enseignant de la classe. La phase de saisie des premières versions des textes d'élèves par

\footnotetext{
13 Ainsi les connotations péjoratives parfois attachées au mot travail trouvent une explication dans l'origine latine du mot, le tripalium étant un instrument de torture. Cet élément d'érudition trouve un accueil amusé et intéressé d'élèves de cycle 3 qui appréhendent de cette manière les enjeux de l'étymologie.
} 
l'expérimentatrice a favorisé la relecture autocritique du texte initial pour lui apporter les améliorations voulues : emprunts, ajouts, substitutions, qui font entrer l'élève dans la dynamique de la génétique textuelle [10].

L'accès au statut de texte imprimé donne vie à ce même texte - et pourtant si différent - et aux mots qui en constituent l'âme et la chair. L'institutionnalisation de l'écrit d'élève en un texte présentant des ressemblances typographiques avec les textes consacrés des écrivains reconnus fait entrer le jeune scripteur dans l'univers de l'expérience partagée des mots.

\section{Annexes}

\section{Annexe A : Expressions idiomatiques}

N'écoutant que son bon cœur - reprend vie - reprend des couleurs - bondit de joie - à pas feutrés - fier comme un paon - folle de joie - plus fort que la mort - pleure de joie - presser le pas - être aux bords des larmes - gagné(e) par la peur.

\section{Annexe B : Pages de dictionnaire des synonymes ${ }^{14}$}
dire
demander, questionner, répondre, s'écrier, rétorquer
aller
s'enfoncer, pénétrer, progresser, poursuivre, accélérer
médusé surpris, étonné, abasourdi, admiratif
pétrifié figé, immobile, statufié

\section{Annexe C : Tableau classé de mots extraits de L'Oiseau d'or}

\begin{tabular}{|c|c|c|c|c|}
\hline & Le frère & La sœur & L'oiseau d'or & $\begin{array}{l}\text { Les dangers } \\
\text { rencontrés }\end{array}$ \\
\hline \begin{tabular}{l}
$Z$ \\
Z \\
\multirow{6}{*}{} \\
$\Omega$ \\
$Z$
\end{tabular} & $\begin{array}{l}\text { le malheureux (garçon), } \\
\text { le frère attentionné, le } \\
\text { frère d'Aziza, ce garçon } \\
\text { généreux }\end{array}$ & $\begin{array}{l}\text { la fille exigeante, la } \\
\text { petite fille } \\
\text { tyrannique, la sœur } \\
\text { d'Aziz, sanglots } \\
\text { déchirants }\end{array}$ & $\begin{array}{l}\text { l'oiseau } \\
\text { mélodieux, } \\
\text { l'oiseau maléfique, } \\
\text { le plumage } \\
\text { chatoyant, le chant } \\
\text { envoûtant }\end{array}$ & $\begin{array}{l}\text { le marécage, le piège, } \\
\text { la tempête, le serpent, } \\
\text { le scorpion menaçant, } \\
\text { la chaleur torride, le } \\
\text { soleil de plomb }\end{array}$ \\
\hline 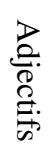 & $\begin{array}{l}\text { courageux, distrait, } \\
\text { inattentif, pétrifié, figé }\end{array}$ & $\begin{array}{l}\text { capricieuse, } \\
\text { téméraire, } \\
\text { affectueuse, } \\
\text { volontaire }\end{array}$ & $\begin{array}{l}\text { rusé, insistant, } \\
\text { opiniâtre, } \\
\text { persévérant }\end{array}$ & \\
\hline 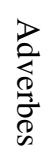 & $\begin{array}{l}\text { spontanément, } \\
\text { affectueusement, } \\
\text { malheureusement }\end{array}$ & Prestement & $\begin{array}{l}\text { mélodieusement, } \\
\text { sournoisement }\end{array}$ & \\
\hline 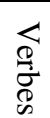 & s'anime, embrasse & $\begin{array}{l}\text { parcourt, rencontre, } \\
\text { aperçoit, craint }\end{array}$ & $\begin{array}{l}\text { chante, questionne, } \\
\text { fige, transforme, } \\
\text { ricane, volète }\end{array}$ & \\
\hline
\end{tabular}

\footnotetext{
14 Chacune de ces annexes correspond à l'une des phases d'une séquence d'apprentissage lexical, qui s'est reproduite à trois reprises pour chaque niveau de classe du cycle 3 de l'école élémentaire.
} 


\section{Annexe D : Questionnaire de choix des aides}

Consignes aux élèves :

1 - Relis ton texte.

2 - Choisis un, deux ou trois outils (parmi les dictionnaires, dictionnaires des synonymes, / mots ou expressions fabriqués par l'enseignant / courts textes d'auteurs).

3 - Mets une croix dans la case correspondant à l'outil que tu as choisi.

\begin{tabular}{|l|l|l|l|}
\hline & le dictionnaire & $\begin{array}{l}\text { la liste des mots et } \\
\text { des expressions }\end{array}$ & des textes d'auteurs \\
\hline J'ai utilisé & & & \\
\hline J'explique mon choix & & & \\
\hline
\end{tabular}

4 - Dis pourquoi.

5 - Ajoute ou supprime des mots ou des expressions en t'aidant de l'outil que tu as choisi. Si nécessaire modifie ensuite les phrases concernées par tes transformations.

\section{Références}

[1] R. Barthes, Le grain de la voix. Entretiens 1962-1980, Paris : Points Seuil (1980)

[2] OCDE, PISA 2009 Results : What Students Know and Can Do - Student Performance in Reading, Mathematics and Science (Volume I) (2010) En ligne : http://www.oecd.org/ pisa/pisaproducts/48852548.pdf

[3] É. Bautier, Mobilisation de soi, exigences langagières scolaires et processus de différenciation, Langage et société, 111, 51-72 (2005)

[4] R. Gagnon \& H. Ziarko, Indicateurs lexicaux dans les textes de comparaison et de problèmesolution produits par des élèves de 8-9 ans, Pratiques, 155-156, 97-107 (2012)

[5] B. Marin, De la place des verbes génériques dans l'écriture scolaire de textes de fiction : de la mise en mots à l'usage de mots précis, La Linguistique. 47(1), 89-176 (2011)

[6] A. Sardier, Favoriser l'accès lexical en production écrite. Pratiques, 155-156, 127-146 (2012)

[7] G. Denhière \& S. Jhean-Larose, L'Acquisition du vocabulaire, Paris : Ministère de l'Éducation nationale - Éduscol (2011)

[8] M. Lewis, Implementing the Lexical Approach : Putting Theory into Practise, Hove UK : Language Teaching Publications (1997)

[9] É. Bautier \& R. Goigoux, Difficultés d'apprentissage, processus de secondarisation et pratiques enseignantes : une hypothèse relationnelle, Revue française de Pédagogie, 148, 89-100 (2004)

[10] A. Grésillon, Éléments de critique génétique. Lire les manuscrits modernes, Paris : Presses universitaires de France (1994) 